\title{
The ASTRI Mini-Array at Teide Observatory
}

\author{
Lucio Angelo Antonelli ${ }^{a, b, *}$ for the ASTRI Project ${ }^{c}$ \\ ${ }^{a}$ INAF-Osservatorio Astronomico di Roma, \\ Via Frascati 33, Monte Porzio Catone (Roma), Italy \\ ${ }^{b}$ ASI-Space Science Data Center, \\ Via del Politecnico s.n.c., Roma, Italy \\ ${ }^{c}$ http://www.astri.inaf.it/ \\ E-mail: angelo.antonelli@inaf.it
}

The ASTRI Mini-Array is an international project led by the Italian National Institute of Astrophysics (INAF) with the participation of the Instituto de Astrofisica de Canarias, Brazil and South Africa to be installed at the Teide Observatory, in Tenerife, to observe the Very High Energy sky in the range of a few $\mathrm{TeV}$ up to $100 \mathrm{TeV}$ and beyond. The ASTRI Mini-Array technology is based on the ASTRI-Horn prototype, a small-sized Cherenkov telescope (SST) developed by INAF within the Cherenkov Telescope Array (CTA) Project and located in Italy at the INAF "M.C. Fracastoro" observing station (Mt. Etna, Sicily). The telescope is characterized by a dual-mirror optical system and curved focal surface covered by a SiPM sensors camera managed by a fast front-end electronics. The ASTRI Mini-Array is composed of nine ASTRI-Horn-like telescopes. It is going to be developed by the ASTRI Collaboration in all aspects from the design, construction and implementation of the entire hardware and software system, including a dedicated off-site Data Center, to the final scientific products. The ASTRI Mini-Array is going to be operated as an observation facility led by INAF and thanks to its sensitivity, especially at energies greater than $1 \mathrm{TeV}$, represents the key instrument to perform very soon a ground breaking achievement in the field of extreme gamma rays, up to $100 \mathrm{TeV}$ and beyond. In this contribution, the project status and the expected performance of the ASTRI Mini-Array will be reviewed.

$37^{\text {th }}$ International Cosmic Ray Conference (ICRC 2021)

July 12 th - 23rd, 2021

Online - Berlin, Germany

\footnotetext{
*Presenter
} 


\section{The ASTRI Project}

The interest of scientists of the Italian National Institute for Astrophysics (INAF) in high energy astrophysics has a very long tradition since the sixties of the past century. Such an interest has been recently confirmed through the participation of INAF scientists in the realization and exploitation of the FERMI and AGILE $\gamma$-ray satellites and of the MAGIC telescopes. The ASTRI project was originally born in 2010 as a "flagship project" funded by the Italian Ministry of University and Scientific Research, led by INAF, and aimed at the technological development of the next generation of Imaging Atmospheric Cherenkov Telescopes (IACT) for ground-based gamma ray astronomy. The acronym ASTRI stands for "Astronomia a Specchi a Tecnologia Replicante Italiana" which means Astronomy with mirrors built through Italian replica technology. At present the ASTRI project involves more than 150 researchers of the INAF institutes in Rome, Milan, Padua, Bologna, Teramo, Palermo and Catania. The Universities of Perugia, Padova, Catania, Genova and the Milano Polytechnic together with the INFN sections of Roma Tor Vergata and Perugia participate to the project and also the Italian Space Agency is participating through its Space Science Data Center in Rome. The University of Sao Paulo in Brazil, the North Western University in South Africa and the Instituto de Astrofisica de Canarias in Spain are the international partners of the project. Finally, several Italian and foreign industrial companies actively participate to the realization of the project.

\subsection{The ASTRI Prototype}

The ASTRI project has been conceived as an end-to-end project divided in two different phases [1]. The first phase of the project has consisted in the design, realization and deployment of a technological demonstrator represented by a prototype of the 4 meters class telescopes to be tested in an astronomical site. This prototype has been the first Cherenkov telescope to adopt a dual mirror optical configuration and among the first to use Silicon Photo-Multipliers (SiPM) as detectors. The end-to-end approach has required to realize the full chain from images acquisition to their analysis which comprehend not only the telescope and the camera but also the internal and external calibration system, the hardware and control software, the data reduction and data analysis software and the archive system. The telescope was installed in 2014 at the M.G. Fracastoro station of the INAF - Catania Astrophysical Observatory, placed at an altitude of 1725 meters above the sea level inside the Etna regional park. The validity of the innovative design has been successfully demonstrated with the detection of the Crab nebula at energies larger than 3.5 TeV in December 2018 [2].

\subsection{The ASTRI Mini-Array}

The second phase of the ASTRI project is represented by the development of a Mini-Array composed of nine dual-mirror IACTs, based on the evolution of the ASTRI-Horn prototype. In fact, the new telescopes design is implementing a larger field of view (more than 10 degrees in diameter) and is equipped with the updated version of the compact ASTRICAM camera, based on Hamamatsu silicon photomultipliers and on the CITIROC-1A ASIC developed by Weeroc in collaboration with INAF for the read-out electronics. The ASTRI Mini-Array is going to be installed at the Teide Astronomical Observatory, operated by the Instituto de Astrofisica de Canarias (IAC), on Mount Teide, in Tenerife (Canary Islands). The ASTRI MA will be operated by INAF in collaboration 


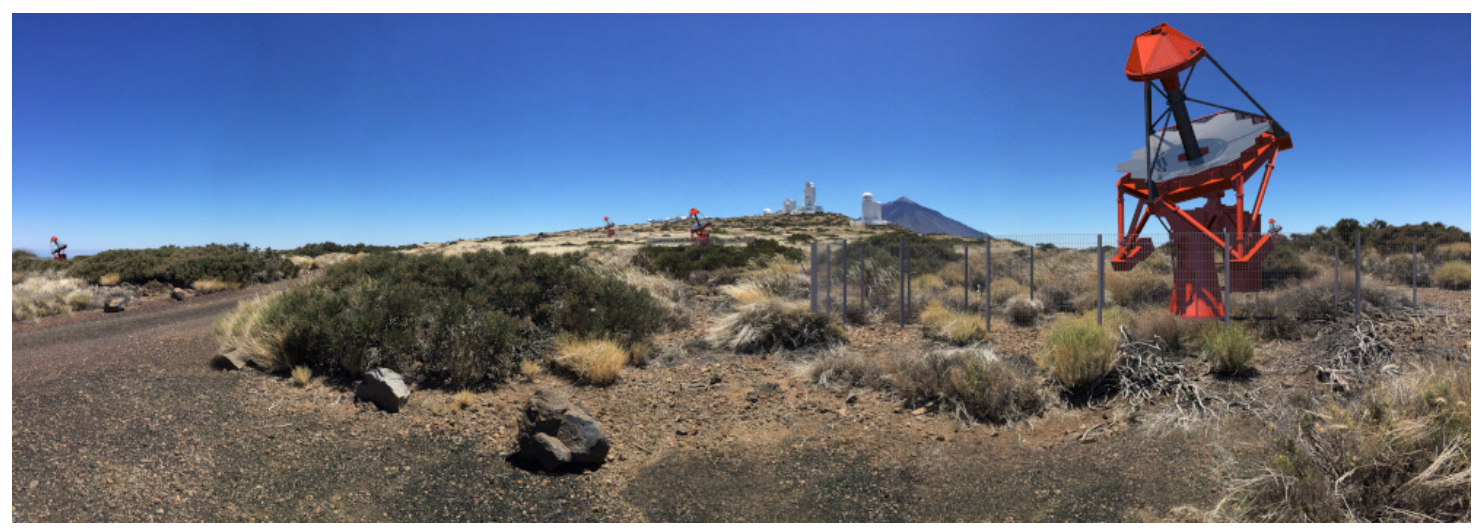

Figure 1: Artistic view of ASTRI Mini-Array Telescopes. From left to right: ASTRI-2, ASTRI-1, ASTRI-4, ASTRI-7 in the foreground surrounded by the fence, and ASTRI-6 in the background behind ASTRI-7. The picture shows an unrealistic scenario with telescopes pointing at the Sun. Credits: M. Leone.

with IAC. Thanks to its nine telescopes the ASTRI mini-array, compared to currently operating IACT systems[3], will extend the sensitivity up to $100 \mathrm{TeV}$ and beyond, an almost never explored energy range by IACTs, being already a factor of two more sensitive at energies larger than $20 \mathrm{TeV}$ for 50 hours of observations. Moreover, it will benefit from a much larger field of view, which will allow to monitor simultaneously a few close-by sources during the same pointing. The combination of the sensitivity extended to $100 \mathrm{TeV}$ and of the homogeneous performance across the FOV will allow to study e.g. emission from extended sources such as SNRs and PWNs at E $>10 \mathrm{TeV}$, and to investigate the presence of spectral cut-offs. The energy threshold about $2 \mathrm{TeV}$ will naturally lead to focusing the schedule on a number of well defined deeply exposed, science driven targets. Finally, the ASTRI mini-array will operate when the present IACT systems, observing in a lower but partly overlapping energy range, will still be active, allowing direct comparison of scientific data (spectra, light-curves, integral fluxes). Also, fruitful synergies with HAWC and possibly with LHAASO, surveying a very large stripe of the northern sky, with pointed observations are also clearly foreseen. In summary, the ASTRI mini-array will allow to carry out seminal studies on both Galactic[4] and extra-Galactic[5] sources, tackling frontier issues at the intersection of the fields of astrophysics, cosmology, particle physics and fundamental physics [6] as well as transient sources [7]. Figure 1 shows an artistic view of what part of the ASTRI Mini-Array will look like after construction.

\section{The ASTRI Mini-Array at the Teide Observatory}

The ASTRI Mini-Array infrastructures are distributed among different locations in Tenerife, La Palma and Italy. The ASTRI Mini Array Site at the Teide Observatory will host the telescope array, other equipment (e.g. weather stations, LIDARs etc.) and site support facilities (e.g. control room, on-site data centre, power management system etc.). In particular, the on-site control room will be hosted at the THEMIS telescope and the on-site data centre at the Residencia of the Teide Observatory. The ASTRI Mini Array Support Site located at IAC premises in La Laguna, hosting the remote control room, and warehouse spaces. La Palma will host administrative services at the Fundacion Galileo Galilei. Finally Italy will host the off-site data centre at the INAF Astronomical 
Observatory in Rome and, eventually, remote control rooms in other INAF institutes. The final layout of the ASTRI mini-array at the Teide Observatory is shown in Figure 2. The figure shows the positions of the 9 telescopes, of the 2 meteorological stations, of the LIDAR, of the control room and of the data centre. The ASTRI Mini-Array layout was defined in order to maximize scientific performance. However, the characteristics of the area, the possibility to exploit available site infrastructures, the safety of operations and the impact on the activities of Teide observatory were also considered. The number of telescopes of the ASTRI Mini-Array guarantees a sensitivity better than existing facilities already at $10 \mathrm{TeV}$ in the northern hemisphere and at $15 \mathrm{TeV}$ in the case of the southern ones [8]. The position of the telescopes and their spacing has been analysed using Monte Carlo simulations. The simulations show that a symmetric layout with a spacing of about 250 meters yields the better compromise between a very good performance at high energies $(>10 \mathrm{TeV})$ and a good sensitivity at lower ones. Then the final layout has been chosen as a compromise among the necessity to preserve symmetry and optimum spacing, the shape of the available area and the possible constraints on the position of telescopes. The layout is asymmetrical being more elongated in the North-East/South-West direction with a median spacing among neighbouring telescope of about $200 \mathrm{~m}$. Nevertheless, the variation in performance due to the asymmetry are not relevant in the whole energy range. In the development of the layout we also took advantage of already existing infrastructures in order to both minimize the impact on the ecosystem of the area and on the activities of the other scientific facilities at the Teide observatory.

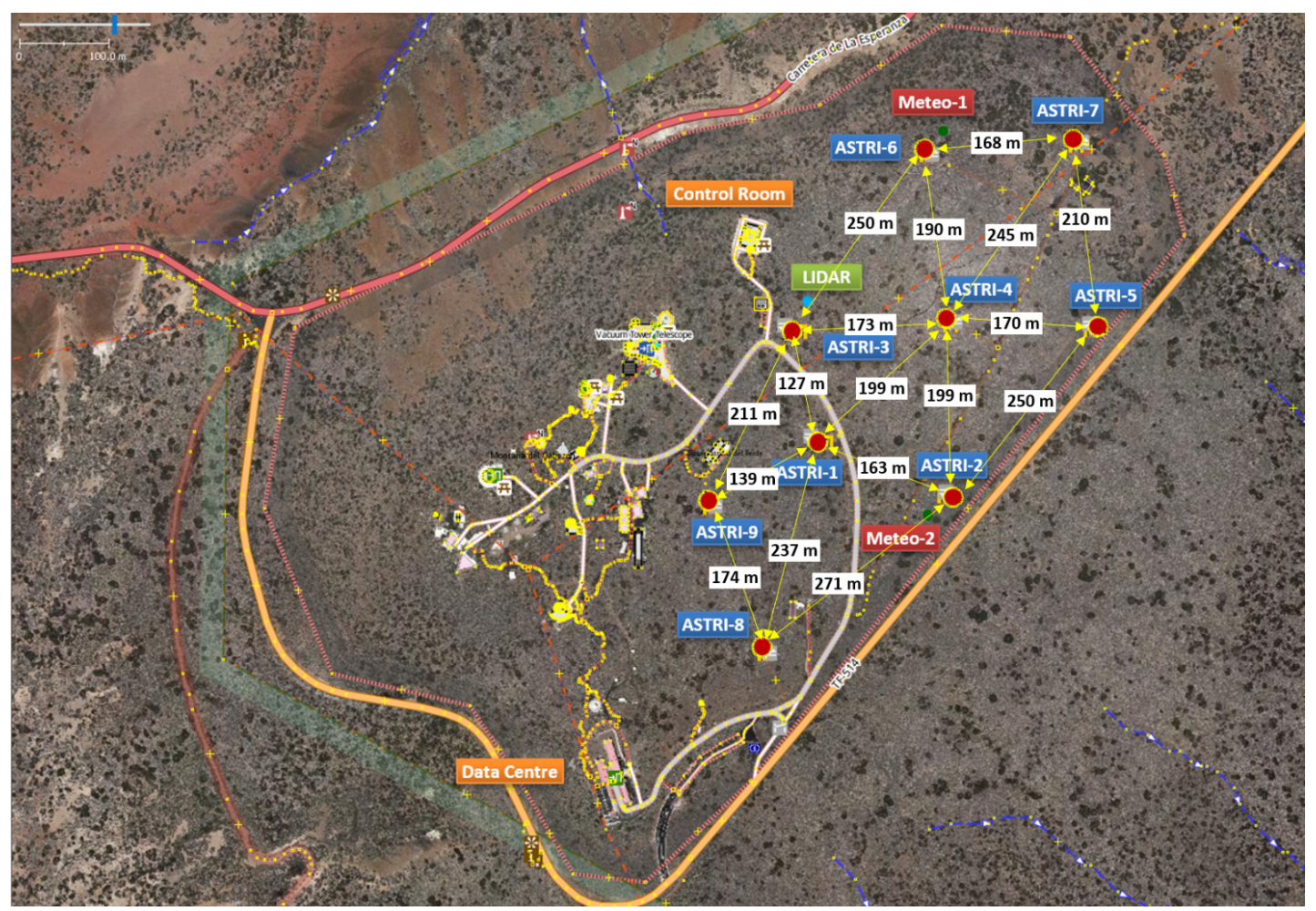

Figure 2: The final layout for the ASTRI mini-array 


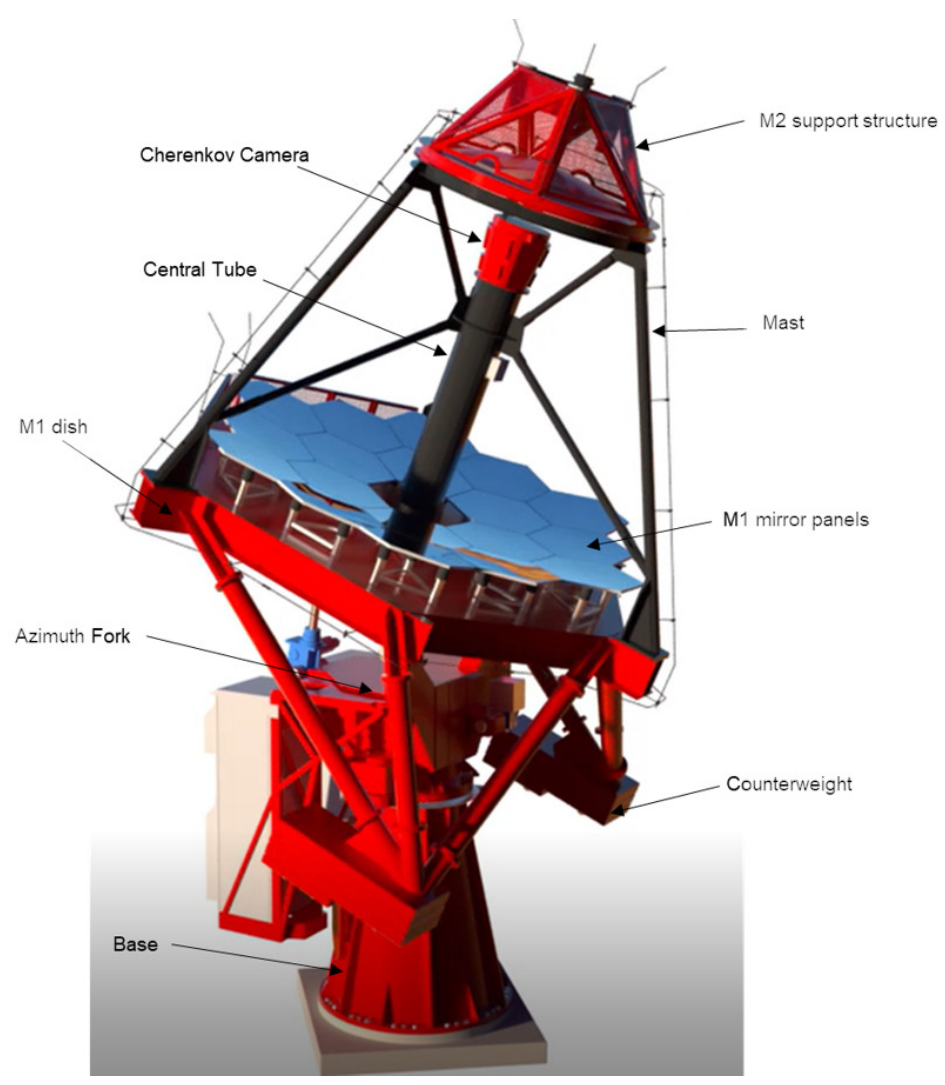

Figure 3: 3D model of a telescope of the ASTRI mini-array. Some of the opto-mechanical components are explicitly listed

\section{The ASTRI Telescopes}

The "Telescope System" includes all the hardware and software components needed to collect and image Cherenkov light from air showers and star light for intensity interferometry but also the auxiliary assemblies needed to support this function. Figure 3 shows a 3D model of one of the ASTRI telescopes to be deployed at the Teide Observatory site.

The main subsystems of each ASTRI telescope forming the Mini-Array are: Mechanical Structure, Optics, Cherenkov Camera, Intensity Interferometry Module, Auxiliary assemblies. The mechanical structure of the ASTRI telescope adopts an alt-azimuthal design. The primary mirror dish, which supports the primary mirror, is mounted on an azimuth fork, which allows rotation around the elevation axis from 0 to +91 degrees. The mast structure, that supports the secondary mirror and the camera, is placed on the primary mirror dish. Exploiting the lessons learned with the prototype the electro-mechanical structure has been optimized in terms of mass, functionality and maintainability. In particular the mass has been reduced by $30 \%$ going from 25 tons down to 17.5 tons while keeping the stiffness of the structure. This allowed the removal of the primary active control system. Modifications to the secondary mirror support structure and to the primary mirror dish structure brought to easier maintenance procedures and less vignetting. A detailed description of the improved electro-mechanical structure can be found in [9]. The optical design is based on a modified Schwarzschild-Couder configuration. The primary mirror (M1) is segmented while the 
secondary (M2) is monolithic. The primary is composed by a set of 18 hexagonal-shaped panels. The profiles of both mirrors are aspheric with substantial deviations from the main spherical component. The primary mirror has a diameter of $4.3 \mathrm{~m}$ while the secondary mirror diameter is $1.8 \mathrm{~m}$. The primary-to-secondary distance is $3 \mathrm{~m}$ and the secondary to camera distance $0.52 \mathrm{~m}$. This optical setup delivers a plate scale of $37.5 \mathrm{~mm} /$ degree, an equivalent focal length of $2150 \mathrm{~mm}$ and an effective area of about $5 \mathrm{~m}^{2}$. The optical design of the Mini-Array telescope has remained unchanged but for the coating of the mirrors that was improved using a combination of $\mathrm{Al}, \mathrm{SiO}_{2}$ and $\mathrm{ZrO}_{2}$. The small plate scale resulting from the optical optical design leads to a Cherenkov camera of compact dimensions. This, in turn, allows the use of silicon photomultipliers (SiPM) as focal plane detectors. The SiPM detectors chosen for the ASTRI Mini-Array Cherenkov cameras are those of LVR3 series produced by Hamamatsu photonics. They are uncoated and have linear dimensions of $7 \times 7 \mathrm{~mm}$. To cover the corrected filed of view of the telescope 2368 pixels are needed. The pixels are grouped in matrices of $8 \times 8$ pixels and 37 matrices are arranged to adapt to the curved focal plane of the telescope.

The characteristics of the optical system coupled with the physical characteristics of the SIPM detectors yields an angular pixel size of 0.19 degree and a field of view of 10.5 degrees.

The ASTRI camera read-out electronics ensures a high efficiency detection of a Cherenkov event, with over 1000 events per second, with a very high dynamical range (from 1 to 1500 photoelectrons per pixel). At the hearth of the electronics is the CITIROC-1A ASIC that implements a customized signal shaper and peak detector to acquire the SiPM pulses. The camera trigger is a topological one, activated when a given number of contiguous pixels presents a signal above a threshold equivalent to a given number of photo-electrons.

A detailed description of the characteristics and capabilities of the Cherenkov camera developed for the ASTRI-Horn prototype can be found in [10].

The Monitoring, Characterization, and Calibration System is including all the hardware and software devoted those actions. The Environmental Monitoring System includes all the hardware necessary to monitor the atmospheric weather conditions at the observing site. In particular, two meteorological stations are going to be installed on telescopic towers, solar powered and wi-fi connected to the ASTRI Mini-Array network. Rain sensors and humidity sensors will be mounted on each single telescope. The Atmospheric Characterization System includes all the devices necessary to measure the Night Sky Background (UVSiPM and SQM) and the atmospheric extinction (LIDAR) in the observing direction. These data will be used to properly correct the Cherenkov measurements. Finally, the array calibration system is made by a single device: the illuminator in order to calibrate the ASTRI Telescopes in energy.

\section{The ASTRI Mini-Array Software System}

The ASTRI Mini-Array software system will provide a set of tools to the user from the preparation of an observing proposal, through the execution of the observations, to the analysis of the acquired data and the storage and retrieval of all the data products from the archive.

The Mini-Array software system is envisioned to handle an observing cycle, i.e. the end-toend control and data flow system. The observing cycle can be divided into the following main phases:Observation preparation; Observation execution; Data Processing; Data Dissemination. 


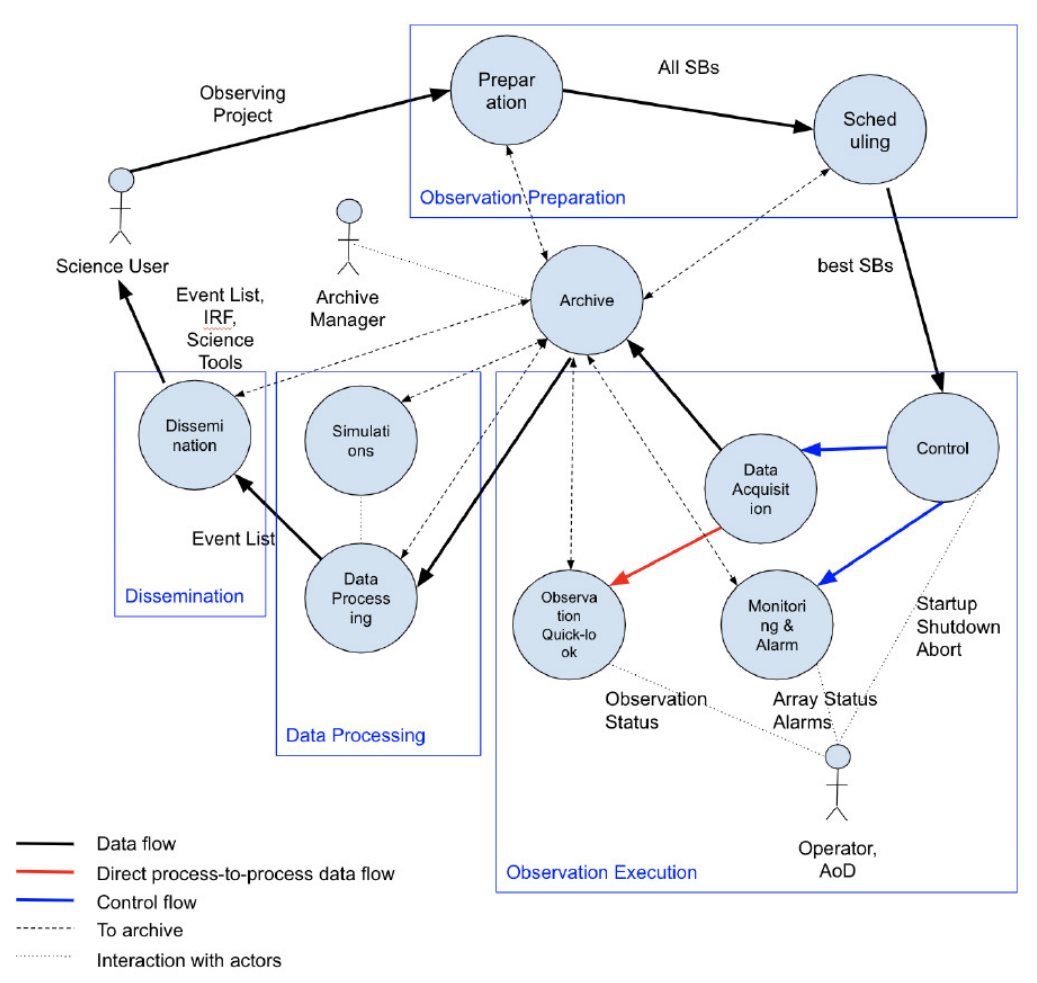

Figure 4: MA Software System data and information flow (schematic) with the four main phases. The outer solid black and red lines show the logical data flow, where the solid blue lines are control flow. Direct process-to-process communication is indicated with a red line. The dashed lines directed to/from the Archive indicate that a) all data are saved and can be retrieved from the Archive, and b) that the physical data flow may be handled by the Archive.

Figure 4 shows how the data and information produced flow during an observing cycle. The central role of the archive is clear. The software system devoted to control all the operations carried out at the MA site, including the startup of the MA system, is the so called Supervisory Control And Data Acquisition (SCADA) Software. This is a central control system which interfaces and communicate with all equipment and dedicated software installed On-Site. It is also interfacing with the firmware and low-level software dedicated to the low-level hardware control operations. The software service providing storage and organization for all data, data products, and metadata generated for and by the Mini-Array, and defined by the Mini-Array Data Models is the ASTRI Archive System. The ASTRI Data Processing System is the software system used to calibrate and reduce acquired date. This software is also used to check the quality of the final data products. The Science Support System is the software system which provides the main point of access for the exchange of science-related data and information with the ASTRI Science Users, and which supports the whole science-related workflow, from the Observing Project submission to the access to the archived high-level Mini-Array science data products and the corresponding Science Tools to support data analysis. The Simulations System is the software system that runs Monte Carlo simulations to provide simulated data for the development of reconstruction algorithms and for the characterization of real observations. 
Acknowledgements This work was conducted in the context of the ASTRI Project. This work is supported by the Italian Ministry of University and Research (MUR) with funds specifically assigned to the Italian National Institute for Astrophysics (INAF). We acknowledge support from the Brazilian Funding Agency FAPESP (Grant 2013/10559-5) and from the South African Department of Science and Technology through Funding Agreement 0227/2014 for the South African Gamma-Ray Astronomy Programme. This work has been supported by H2020-ASTERICS, a project funded by the European Commission Framework Programme Horizon 2020 Research and Innovation action under grant agreement n. 653477. IAC is supported by the Spanish Ministry of Science and Innovation (MICIU). We also thank the Fundación Galileo Galilei - INAF, Fundación Canaria (FGG) for its support in deploying and managing the ASTRI Mini-Array in Tenerife.

\section{References}

[1] S. Scuderi, The ASTRI Program, in European Physical Journal Web of Conferences, vol. 209 of European Physical Journal Web of Conferences, p. 01001, Sept., 2019, DOI.

[2] S. Lombardi, O. Catalano, S. Scuderi, L.A. Antonelli, G. Pareschi, E. Antolini et al., First detection of the Crab Nebula at TeV energies with a Cherenkov telescope in a dual-mirror Schwarzschild-Couder configuration: the ASTRI-Horn telescope, 634 (2020) A22 [1909.12149].

[3] S. Vercellone, The ASTRI Mini-Array Core Science Program, in Proceedings of 37th International Cosmic Ray Conference - PoS(ICRC2021), vol. 395, p. 896, 2021, DOI.

[4] A. Dai, E. Amato, A. Burtovoi, A. Compagnino, M. Fiori, A. Giuliani et al., Observatory Galactic Science with the ASTRI-Mini Array during the observatory phase of the project, in Proceedings of 37th International Cosmic Ray Conference - PoS(ICRC2021), vol. 395, p. 900, 2021, DOI.

[5] F.G. Saturni, L.A. Antonelli, C. Arcaro, B. Balmaverde, J. Becerra Gonzalez, A. Caccianiga et al., Extragalactic Observatory Science with the ASTRI Mini-Array at the Observatorio del Teide, in Proceedings of 37th International Cosmic Ray Conference - PoS(ICRC2021), vol. 395, p. 888, 2021, DOI.

[6] M. Cardillo, "The ASTRI Mini-Array: a breakthrough in the Cosmic Ray study", in Proceedings of 37th International Cosmic Ray Conference - PoS(ICRC2021), vol. 395, p. 807, 2021, DOI.

[7] A. Stamerra, F.G. Saturni, J.G. Green, L. Nava, F. Lucarelli and L.A. Antonelli, TeV Transients with the ASTRI Mini-Array: a case study with GRB 190114C, in Proceedings of 37th International Cosmic Ray Conference - PoS(ICRC2021), vol. 395, p. 890, 2021, DOI.

[8] S. Lombardi, L.A. Antonelli, C. Bigongiari, M. Cardillo, S. Gallozzi, J.G. Green et al., Performance of the ASTRI Mini-Array at the Observatorio del Teide, in Proceedings of 37th International Cosmic Ray Conference - PoS(ICRC2021), vol. 395, p. 884, 2021, DOI.

[9] G. Marchiori, A. Busatta, E. Marcuzzi, C. Manfrin, I. Folla, G. Pareschi et al., ASTRI SST-2M: the design evolution from the prototype to the array telescope, in Ground-based and Airborne Telescopes VII, H.K. Marshall and J. Spyromilio, eds., vol. 10700 of Society of Photo-Optical Instrumentation Engineers (SPIE) Conference Series, p. 107005W, July, 2018, DOI.

[10] O. Catalano, M. Capalbi, C. Gargano, S. Giarrusso, D. Impiombato, G. La Rosa et al., The ASTRI camera for the Cherenkov Telescope Array, in Ground-based and Airborne Instrumentation for Astronomy VII, C.J. Evans, L. Simard and H. Takami, eds., vol. 10702 of Society of Photo-Optical Instrumentation Engineers (SPIE) Conference Series, p. 1070237, July, 2018, DOI. 